INRA Prod. Anim.,

1988,1 (2), 83-92

\section{Y. COGNIÉ}

INRA Nouzilly

Station de Physiologie

de la Reproduction

37380 Monnaie

\title{
Nouvelles méthodes utilisées pour améliorer les performances de reproduction chez les ovins
}

\author{
Pour maitriser la reproduction des ovins, l'éleveur dispose d'un éventail de \\ méthodes depuis la mise en œuvre de pratiques simples - par exemple, \\ introduire un bélier dans un troupeau de brebis stimule leur fonctionnement \\ ovarien - jusqu'à l'utilisation de techniques sophistiquées comme par exemple \\ le transfert d'embryons après un traitement superovulant.
}

\begin{abstract}
Les techniques de reproduction de plus en plus précises appliquées à la production ovine reflètent directement la progression rapide des connaissances en physiologie de la reproduction. Léquilibre des mécanismes contrôlant la reproduction repose sur une relation permanente entre les centres nerveux supérieurs et les gonades, relation assurée par des hormones glycoprotéiques ou stéroïdiennes. Les centres nerveux comportent deux étages, le premier (hypothalamus) contrôlant l'activité du second (hypophyse) par l'intermédiaire de la gonadolibérine (GnRH). Chez la femelle, les hormones
\end{abstract}

\section{Résumé}

Les périodes d'anœstrus (saisonnier ou post-partum) et le faible taux d'ovulation limitent l'efficacité de la reproduction chez la brebis. Cet article résume les recherches récentes effectuées dans le but d'améliorer les paramètres de la reproduction dans différents systèmes de production.

Plusieurs méthodes sont disponibles pour synchroniser les chaleurs. Le choix de la méthode doit dépendre du système de lutte utilisé et du niveau de prolificité souhaité par l'éleveur. Les avantages découlant de l'utilisation de la technique associant un progestagène à PMSG sont présentés. Cette technique permet avec succès la mise en place de l'insémination artificielle, mais une réduction de la variabilité du moment d'ovulation doit être recherchée. Afin d'éliminer les problèmes liés aux difficultés de passage des spermatozoïdes à travers le cervix, l'insémination utérine sous contrôle cœlioscopique est proposée, surtout lorsque de la semence congelée est utilisée. L'induction d'ovulations fertiles pendant l'anœstrus saisonnier peut également être obtenue en utilisant "l'effet bélier" associé à un traitement progestagène et/ou à des manipulations de la photopériode ou de la mélatonine.

Le taux de prolificité peut être augmenté par injection de gonadotropines (PMSG ou pFSH) ou immunisation contre les stéroides. Limmunisation active contre l'androsténedione permet d'obtenir entre 18 et $40 \%$ d'agneaux nés en plus chez les brebis traitées de différents génotypes et dans différents systèmes de conduite des troupeaux. Chez la brebis charmoise immunisée pendant 4 années successives à l'automne, un gain moyen de 0,31 agneau sevré a été obtenu.

L'importance des pertes embryonnaires précoces, d'origine non génétique, peut être minimisée par l'apport de progestérone exogène ou la stimulation de la sécrétion endogène de progestérone par les gonadotropines. L'estimation du nombre de fœetus effectuée en milieu de gestation par échographie permet d'adapter les rations des mères à leurs besoins et de réduire la mortalité périnatale des agneaux. hypophysaires stimulent la croissance terminale des follicules (FSH), la maturation des ovocytes et l'ovulation (LH). En retour, les stéroïdes ovariens agissent, soit au niveau hypothalamique pour freiner la sécrétion du GnRH, soit au niveau hypophysaire pour contrôler la sécrétion de LH et FSH (Figure 1). Le liquide contenu dans la cavité des follicules ovariens est riche en composés encore mal caractérisés. Parmi ces composés, on sait que l'inhibine freine la sécrétion de FSH par l'hypophyse et que d'autres messagers interviennent au niveau des échanges intra-folliculaires ou au niveau des relations entre différents follicules.

Lallongement de la durée du jour induit chez la brebis une plus grande sensibilité hypothalamique à la rétroaction négative des stéroïdes et une diminution de la sécrétion nocturne de mélatonine par la glande pinéale. Le ralentissement de l'activité gonadotrope et l'arrêt des cycles sexuels sont la conséquence de ces changements. A l'inverse, l'accélération de la fréquence de décharge de l'hormone hypothalamique et de la $\mathrm{LH}$ chez les femelles mises en présence de la phéromone mâle ("effet bélier") ou soumises à une photopériode décroissante (augmentation de la sécrétion nocturne de mélatonine) permet l'induction d'ovulation.

Outre la photopériode et l'environnement "social", d'autres facteurs de l'environnement ont des effets sur la reproduction. L'influence de la nutrition, de la température et des conditions d'élevage (stress) sur le taux d'ovulation, la fertilité et la survie embryonnaire a été étudiée (Ortavant 1985).

Il est difficile de faire une revue synthétique des techniques permettant de mieux maîtriser la reproduction étant donné la variété des méthodes disponibles et des objectifs à àtteindre :

- ajustement de la répartition annuelle des besoins alimentaires aux disponibilités (élevages extensifs de l'hémisphère Sud ayant un faible revenu par tête de bétail). 


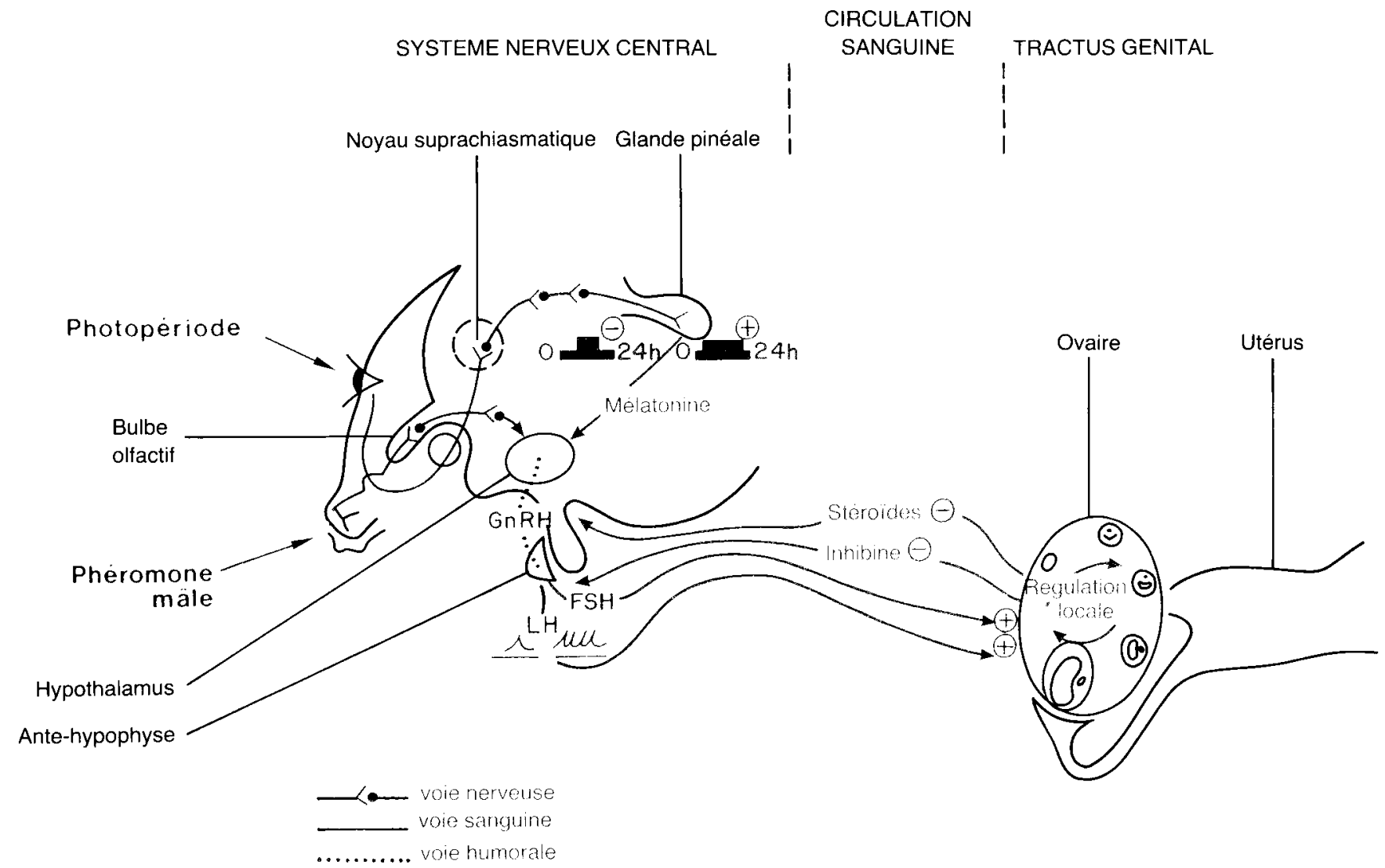

Figure 1. Modèle schématique de régulation de la reproduction chez la brebis.
- augmentation de la taille de la portée et de la fréquence des agnelages (élevages intensifs de l'hémisphère Nord bénéficiant de prix de vente plus élevés).

- accélération de l'amélioration génétique d'une population pour augmenter la production de lait, de viande ou de laine.

Quels que soient les objectifs fixés, l'amélioration des performances de reproduction permet d'augmenter le niveau de production sans accroître le nombre de femelles dans le troupeau. Plusieurs stratégies ont été développées dans ce but, nous allons en présenter quelques unes, dont les plus récentes sont encore du domaine des laboratoires de recherches.

\section{1 / Utilisation des techniques hormonales pour la synchronisation et/ou l'induction des chaleurs}

La maîtrise hormonale du cycle sexuel est basée sur l'association d'un blocage du développement folliculaire et de l'ovulation par la progestérone (Dutt et Casida 1948) avec l'administration de gonadotropines stimulant la croissance terminale des follicules à la fin de cette perriode de blocage.

\section{1 / Choix du traitement progestagène}

Pour contrôler la reproduction chez la brebis, il est important de bien maîtriser le moment d'ovulation afin de réaliser l'insémination artificielle (IA) ou la monte naturelle à un moment prédéterminé sans avoir à détecter les chaleurs. Des substances de synthèse analogues à la progestérone mais 10 à 20 fois plus actives, telles que l'acétate de médroxyprogestérone (MAP) et l'acé- tate de fluorogestone (FGA) sont administrées à l'aide d'éponges vaginales (Robinson 1964) pour bloquer l'ovulation. Le type de traitement ne semble pas avoir d'effet significatif sur le taux d'ovulation, mais par contre, des différences ont été observées entre traitements sur le moment d'apparition des chaleurs, les taux de fertilité et de prolificité. Le Norgestomet appliqué au moyen d'implants sous-cutanés de $3 \mathrm{mg}$ est métabolisé plus rapidement que le FGA déposé sur des éponges vaginales et le moment moyen d'ovulation observé après la fin du traitement Norgestomet est plus précoce qu'après FGA (55 vs 62 heures, $P<0,05$, Cognié et Mauléon 1983). En avançant le moment d'insémination (50 heures après Norgestomet au lieu de 55 heures après FGA), le nombre d'agneaux nés par brebis inséminée n'est pas différent pour ces deux traitements (Cognié et Mauléon 1983). La synchronisation des chaleurs est plus précise après FGA qu'après MAP (Pearce et al 1984). Ceci se traduit par l'obtention de taux de fertilité similaire lorsque les brebis sont luttées naturellement mais par contre un avantage significatif apparaît en faveur des éponges FGA lorsque l'IA systématique est utilisée (Gordon 1983).

Plus récemment, un dispositif intravaginal appelé CIDR (controlled internal drug release) et contenant $12 \%$ de progestérone a été comparé en Nouvelle Zélande aux éponges FGA avec des résultats favorables (Welch 1983). En France, où plus de $15 \%$ des brebis sont traitées chaque année, la technique des éponges vaginales $40 \mathrm{mg}$ de FGA pendant 12 à 14 jours en saison sexuelle et 30 mg pendant 10 à 12 jours à contre-saison) est de loin la méthode la plus répandue actuellement.

\section{2 / Choix de la dose de PMSG (pregnant mare serum gonadotropine)}

L'injection de PMSG à la fin du traitement progestagène, stimule la croissance folliculaire, avance le début 
Tableau 1. Taux de fertilité et taux de prolificité observés en fonction du nombre de corps jaunes chez la brebis Mérinos d'Arles traitée avec PMSG.

\begin{tabular}{|c|c|c|c|c|}
\hline $\begin{array}{c}\text { Nombre de } \\
\text { corps jaunes } \\
\text { par brebis }\end{array}$ & $\begin{array}{c}\text { Nombre } \\
\text { de } \\
\text { brebis }\end{array}$ & $\begin{array}{c}\text { Taux } \\
\text { de fertilité } \\
(\mathrm{F})\end{array}$ & $\begin{array}{c}\text { Taux } \\
\text { de prolificité } \\
(\mathrm{P})\end{array}$ & $\begin{array}{c}\text { Agneaux nés } \\
\text { par femelle } \\
(\mathrm{F} \times \mathrm{P})\end{array}$ \\
\hline 1 & 185 & 0.64 & 1.00 & 0.64 \\
2 & 213 & 0.74 & 1.62 & 1.20 \\
3 & 32 & 0.71 & 2.44 & 1.73 \\
$\geqslant 4$ & 18 & 0.72 & 2.15 & 1.55 \\
\hline
\end{tabular}

des chaleurs et augmente le taux d'ovulation (Cognié et al 1970). La dose de gonadotropine injectée doit être ajustée précisément en fonction de la saison, de l'état physiologique (brebis allaitante ou tarie) et de la race (Thimonier et Cognié 1977). Chez les races à faible niveau de prolificité, l'induction par PMSG d'ovulations doubles ou triples, améliore la taille de la portée mais également le taux de fertilité chez les femelles polyovulantes (tableau 1). Les brebis naturellement prolifiques étant plus sensibles à PMSG (Bindon et al 1986), les résultats obtenus chez les brebis Romanov (Cornu et Cognié 1984) et les croisées Finnoises (Ainsworth et Shrestha 1985) montrent qu'il est nécessaire, chez ces femelles, de diminuer les doses de gonadotropine administrées.

\section{3 / Avantages de l'association progestagène + PMSG}

\section{a / En lutte naturelle, une gestion du troupeau plus efficace}

La synchronisation des chaleurs et le regroupement des agnelages dans une période courte, à date prévue, peut offrir des avantages :

possibilités d'ajuster l'alimentation (flushing, distribution de compléments en fin de gestation).

- surveillance de l'agnelage permettant de réduire la mortalité des agneaux et des brebis.

- mises en lots homogènes d'agneaux facilitant l'allaitement artificiel, l'élevage, le sevrage et la vente.

\section{$b$ / Insémination artificielle}

En France, l'IA concerne $4 \%$ des brebis. La moitié sont inséminées en dehors de leur saison de reproduction lorsque la libido des béliers est faible, l'autre moitié pendant la saison sexuelle, pour optimiser l'utilisation des béliers issus de schémas de sélection efficaces.

LIA est pratiquée après traitement FGA-PMSG utilisant $400.10^{\circ} \mathrm{spz}$, déposés $55 \pm 1 \mathrm{~h}$ après le retrait de l'éponge chez les brebis taries et $52 \pm 1 \mathrm{~h}$ chez les agnelles. Le sperme peut être conservé pendant $10 \mathrm{~h}$ à $15^{\circ} \mathrm{C}$ dans une solution de lait de vache écrémé (Colas 1983). Pendant la saison de reproduction, les centres d'IA en France obtiennent en routine une fertilité de 65 à $75 \%$ (Colas et Guérin 1979). Les anomalies du sperme augmentent en période de jours croissants et la fertilité des troupeaux inséminés au printemps est réduite sauf si l'on a identifié et éliminé les éjaculats de mauvaise qualité. Le sperme de moindre qualité diminue directement le taux de fécondation et indirectement la taille de la portée en augmentant la mortalité embryonnaire (Colas et al 1985). Ainsi, dans la plupart des centres utilisant l'IA au printemps (et en été), les béliers sont maintenus dans des conditions de photopériode inversée (Colas et al 1984).

Le taux de fécondation est plus élevé après IA systématique chez les brebis détectées en chaleur $36 \pm 6$ heures qui ont un pic de LH apparaîssant entre 36 et 48 heures après le retrait de l'éponge (tableau 2).

Insémination artificielle par voie cervicale nomale : la semence est déposée à l'entrée du col de l'uténus. Pour améliorer le taux de réussite de l'insémination, les spermatozoïdes peuvent être déposés dans la lumière utérine, sous observation calioscopique.

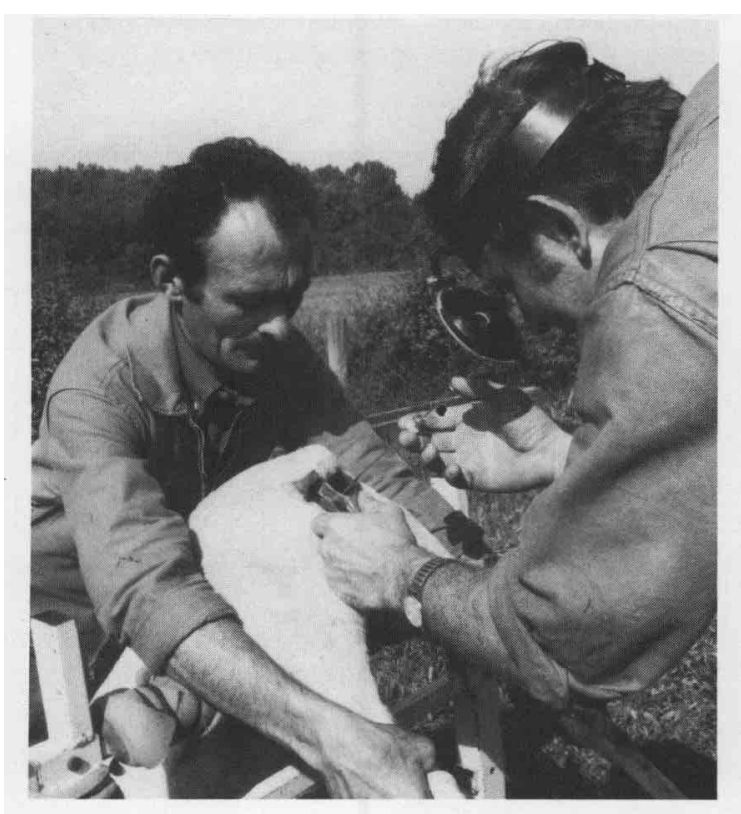

L'administration de progestagène permet de bloquer

l'ovulation, qui est ensuite provoquée par injection de gonadotropine.

Tableau 2. Influence du moment d'apparition du pic de LH sur le taux de fertilité obtenu après IA systématique effectuée $55 \pm 2$ heures après la fin du traitement FGA-PMSG. (D'après Brice et al 1984).

\begin{tabular}{|l|ccccccc|}
\hline & \multicolumn{7}{|c|}{$\begin{array}{c}\text { Brebis ayant un pic de LH débutant } \\
\text { (heures après le retrait de l'éponge) }\end{array}$} \\
& 32 & 36 & 40 & 44 & 48 & 52 & $>52$ \\
\hline $\begin{array}{l}\text { Nombre de brebis } \\
\text { inséminées }\end{array}$ & 40 & 64 & 73 & 72 & 41 & 37 & 59 \\
Taux de fertilité & 0.20 & 0.59 & 0.57 & 0.54 & 0.53 & 0.21 & 0.15 \\
\hline
\end{tabular}


Chez la brebis traitée avec un progestagène, le nombre de spermatozoïdes arrivant dans les oviductes est diminué et la disparition des gamètes mâles dans le tractus génital femelle est plus rapide que chez la brebis non traitée (Quinlivan et Robinson 1969). Ceci implique que lorsque l'IA par voie cervicale est utilisée, elle doit avoir lieu très précisément 6 à 8 heures avant le moment moyen d'ovulation.

La possibilité de contrôler le moment de la décharge préovulatoire, et par conséquent le moment d'ovulation, en utilisant des analogues du GnRH (gonadotrophinreleasing hormone) a été étudiée (Quirke et al 1979). Malheureusement, I'injection de GnRH 24 ou 36 heures après le retrait des éponges ou au début des chaleurs n'améliore pas le taux de fertilité à l'œstrus induit (Fukui et al 1985).

La difficulté de passage du cervix par les spermatozoïdes chez les ovins peut être contournée en déposant la semence dans la lumière utérine sous observation coelioscopique. La voie chirurgicale permet de diminuer 10 fois la dose de spermatozoïdes nécessaires par rapport à l'IA cervicale. Elle laisse, en outre, une plus grande liberté dans le choix du moment d'insémination (entre 60 et 72 heures après le retrait des éponges) même lorsque de la semence congelée est utilisée (Maxwell 1984).

\section{c / Mise à la reproduction plus précoce}

Le traitement FGA-PMSG est utilisé sur des agnelles de 9-11 mois, ayant atteint un développement corporel suffisant (60 à $65 \%$ du poids vif adulte). Dans la région de Roquefort, cette méthode augmente le pourcentage de première mise-bas à 14 mois et par conséquent la durée de production de lait par les brebis Lacaune. Dans ces systèmes intensifs, où la maîtrise de l'alimentation s'est beaucoup améliorée durant les 20 dernières années, le taux d'agnelage des agnelles Lacaune est passé de
$50 \%$ en 1964 à $88 \%$ en 1982 (Perret et Roussely 1984). L'induction des chaleurs par un traitement progestagène-PMSG des brebis en juin ou des agnelles en août est pratiquée sur $1 / 3$ des élevages de la zone de Roquefort et la proportion augmente chaque année.

\section{d / Production désaisonnée d'agneaux}

La plupart des traitements hormonaux sont effectués au printemps pour tirer profit des cours plus élevés de la viande d'agneaux hors saison. Cela entraîne une variation saisonnière importante de la demande aux centres d'IA (tableau 3).

Après induction des chaleurs, la proportion de brebis mettant bas est de $70 \%$ et la proportion d'agneaux nés par brebis traitée est de $110 \%$. On remarquera que les paramètres de reproduction varient de façon significative avec la saison (tableau 3). La plupart des brebis non gestantes après induction des chaleurs retombent en anœstrus et ceci justifie le recours à un diagnostic précoce de gestation pour remettre ces brebis en lutte le plus rapidement possible.

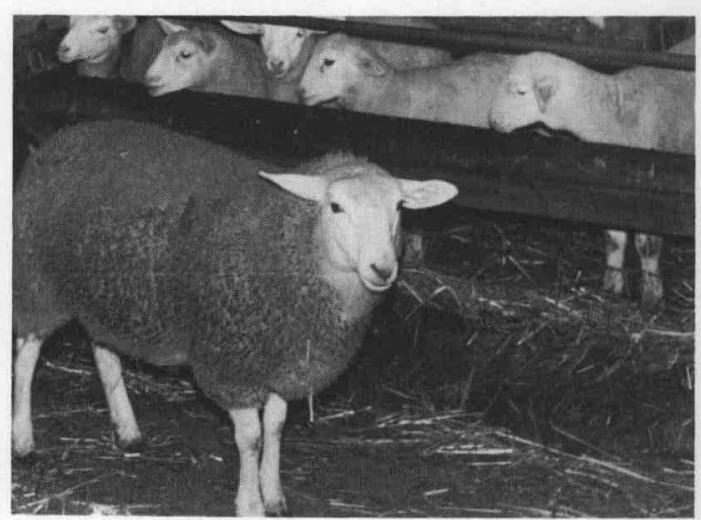

Tableau 3. Variations saisonnières du nombre de brebis inséminées, des taux de fertilité et de fécondité après IA dans le centre de la France. (D'après Colas et al 1984).

\begin{tabular}{|l|c|c|c|c|}
\hline \multirow{2}{*}{} & \multicolumn{4}{|c|}{ Période de l'année (1982) } \\
\cline { 2 - 5 } & Janvier-Mars & Avril-Juin & Juillet-Septembre & Octobre-Décembre \\
\hline $\begin{array}{l}\text { Nombre de brebis } \\
\text { inséminées (\%) }\end{array}$ & $5365(9)$ & $32193(54)$ & $14904(25)$ & $7154(12)$ \\
$\begin{array}{l}\text { Fertilité (en \%) } \\
\text { Nombre d'agneaux nés } \\
\text { pour 100 brebis inséminées }\end{array}$ & 59 & 67 & 74 & 81 \\
\hline
\end{tabular}

Tableau 4. Influence de la durée de l'intervalle entre la mise-bas et la lutte sur la fertilité et la prolificité de brebis Romanov allaitantes après un traitement progestagène-PMSG. (D'après Cornu et Cognié 1984).

\begin{tabular}{|c|c|c|c|c|c|}
\hline $\begin{array}{c}\text { Intervalle } \\
\text { mise-bas lutte } \\
\text { (jours) }\end{array}$ & $\begin{array}{c}\text { Période } \\
*\end{array}$ & (n) & $\begin{array}{c}\text { Fertilité } \% \\
\mathrm{~J} 18^{* *}\end{array}$ & $\begin{array}{c}\text { Fertilité } \% \\
\text { Mise bas }\end{array}$ & Prolificité \\
\hline 50 & B & 60 & 81.7 & 66.7 & 2.70 \\
& A & 78 & $\mathrm{P}<0.01$ & $\mathrm{P}<0.02$ & $\mathrm{P}<0.02$ \\
65 & B & 91 & 61.5 & 41.0 & 2.03 \\
& A & 111 & 92.3 & 75.8 & 3.12 \\
80 & B & 63 & 73.9 & 67.6 & 2.001 \\
& A & 22 & 92.1 & 87.3 & 2.98 \\
\hline
\end{tabular}

- A : anœstrus saisonnier (Mars à Août), B : saison sexuelle (Septembre à Février)

** Estimé par dosage de la progestérone plasmatique 18 jours après la lutte. 


\section{e / Rythme d'agnelage plus rapide}

La présence de l'agneau et l'intensité de la tétée réduisent l'activité ovarienne et le taux de fécondation après induction hormonale des chaleurs post-partum (Hulet et Stormshak 1972). Le sevrage des agneaux le lendemain de la mise-bas augmente le taux de fécondation sur astrus induit de $10 \%$ et de $35 \% 42$ jours après l'agnelage chez des brebis Ile de France allaitant respectivement un ou deux agneaux (Cognié et al 1974). Cependant, excepté dans les pays où le prix de l'agneau est élevé (Canada, Israël), l'allaitement artificiel des agneaux est trop coûteux pour être rentable.

L'accélération du rythme d'agnelage, avec des traitements progestagène-PMSG, peut être obtenue avec des génotypes productifs (croisements Romanov ou Finnois). Par exemple, chez les brebis Romanov en lactation, la survie embryonnaire et le taux d'agnelage ne sont pas significativement diminués quand l'intervalle entre la mise-bas et la gestation suivante est de l'ordre de 50 jours pendant la saison de reproduction et 65 jours en contre-saison (tableau 4).

\section{2 / Avancer la saison de reproduction}

\section{1 / En utilisant "l'effet bélier"}

Dans les systèmes d'élevage intensif, on a tendance à vouloir diminuer les interactions comportementales normales entre mâles et femelles. Cela a pour conséquence de diminuer la capacité de reproduction dans les 2 sexes (Orgeur et Signoret 1985). Les brebis en anœstrus saisonnier ou de lactation, lorsqu'elles ont passé une période isolée des béliers, réagissent à la réintroduction du bélier ("effet bélier") par une ovulation dans les 2 jours qui suivent. L'œstrus a lieu environ 16 jours après, si le corps jaune induit est fonctionnel ou environ 24 jours après si un corps jaune anormal (cycle court) précède un normal (Oldham et Martin 1978). Les mécanismes mis en jeu par l'effet bélier ont été bien étudiés (Martin et al 1986) et les applications pratiques largement développées (Oldham et Pearce 1984, Oldham et Cognié 1980).

Chez des brebis peu saisonnées, l'introduction des béliers stimule le fonctionnement ovarien chez la plupart des femelles, mais le taux de fertilité obtenu est étroitement lié à leur état nutritionnel (Foch et al 1985). L'association d'un traitement progestagène (éponge FGA ou injection i.m. de $20 \mathrm{mg}$ de progestérone) à l'effet mâle permet une augmentation significative du taux de femelles mettant bas, mais pas de la taille de la portée (tableau 5).

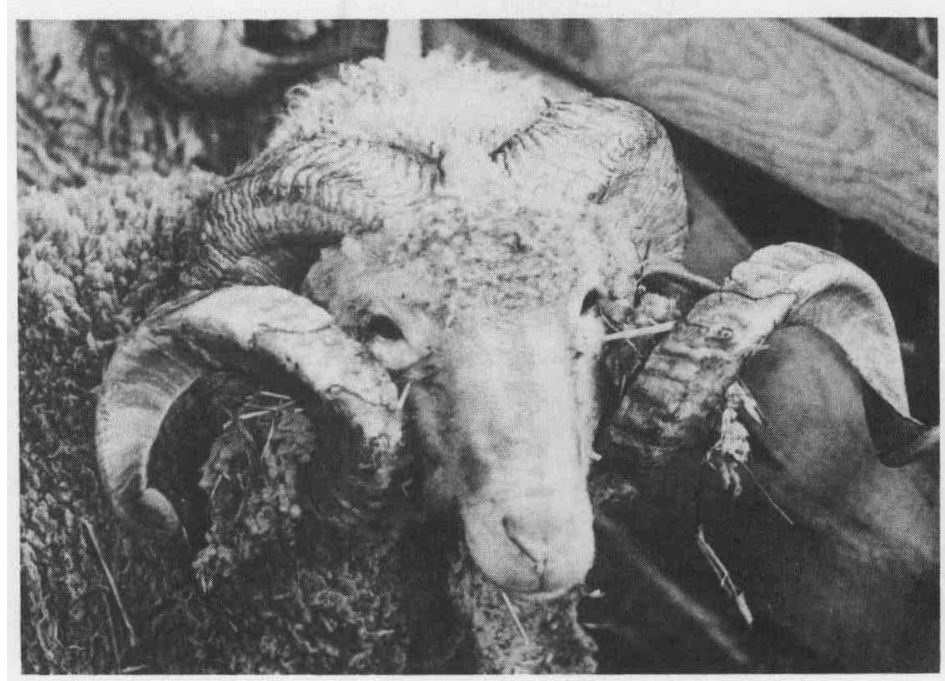

Tableau 5. Comparaison de différentes méthodes pour augmenter les performances de reproduction chez les brebis Mérinos d'Arles et Rasa Aragonesa pendant l'anœestrus saisonnier.

(D'après Folch et al 1985).

\begin{tabular}{|l|c|c|c|c|}
\hline Traitement & (n) & Taux fertilité & Taux prolificité & $\begin{array}{c}\text { Nombre d'agneaux } \\
\text { nés pour } 100 \\
\text { femelles luttées }\end{array}$ \\
\hline Rasa Aragonesa & & & & \\
"Effet mâle" (EM) & 848 & $0.61^{\mathrm{a}}$ & $1.28^{\mathrm{a}}$ & $78^{\mathrm{a}}$ \\
progestérone + EM & 740 & $0.69^{\mathrm{b}}$ & $1.33^{\mathrm{a}}$ & $92^{\mathrm{b}}$ \\
FGA + 500 UI PMSG & 378 & $0.75^{\mathrm{b}}$ & $1.56^{\mathrm{b}}$ & $117^{\mathrm{c}}$ \\
\hline Mérinos d'Arles & 50 & $0.76^{\mathrm{a}}$ & $1.16^{\mathrm{a}}$ & $88^{\mathrm{a}}$ \\
"Effet mâle" (EM) & 48 & $0.90^{\mathrm{b}}$ & $1.30^{\mathrm{a}}$ & $117^{\mathrm{b}}$ \\
FGA + EM & 80 & $0.94^{\mathrm{b}}$ & $1.63^{\mathrm{b}}$ & $153^{\mathrm{c}}$ \\
\hline
\end{tabular}

Pour chaque colonne, les valeurs associées à différentes lettres sont significativement différentes $(\mathrm{P}<0.05)$. (n) Nombre de femelles traitées.

Tableau 6. Influence de l'effet mâle (EM) sur le taux de fertilité obtenu après un traitement progestagènePMSG et IA systématique. (D'après Cognié et al 1984).

\begin{tabular}{|c|c|c|c|}
\hline $\begin{array}{c}\text { FGA-PMSG avec (+) ou } \\
\text { sans (-) EM }\end{array}$ & $\begin{array}{c}\text { Intervalle retrait } \\
\text { éponge-IA (h) }\end{array}$ & $(\mathrm{n})$ & Fertilité (\%) \\
\hline+ & 50 & 50 & $73.5^{\mathrm{a}}$ \\
+ & 55 & 50 & 58.8 \\
- & 55 & 50 & $51.5^{\mathrm{b}}$ \\
\hline
\end{tabular}

a et $\mathbf{b}$ sont significativement différents $(\mathrm{P}<0.05)$. 
Les brebis mises en contact avec le mâle à la fin d'un traitement de synchronisation des chaleurs ovulent plus tôt que les femelles privées de ce contact (Lindsay et al 1975). Dans ce cas, le taux de fertilité obtenu après une insémination artificielle précoce $(50 \mathrm{~h})$ est plus élevé qu'après une IA plus tardive (tableau 6).

\section{2 / En manipulant le signal photopériodique}

Il est possible de lever l'effet inhibiteur des jours longs sur la production de sperme de béliers sélectionnés dans un centre d'IA en réduisant la période d'éclairement. Cette méthode est plus difficile à appliquer sur le troupeau de brebis du fait de la nécessité d'équiper des bâtiments étanches à la lumière.

La mise en évidence d'une période photosensible se situant 16 à 17 heures après l'aube chez le mouton (Ortavant 1977) devrait permettre la simplification des méthodes de manipulation de l'activité sexuelle par la photopériode. Un "flash" lumineux pendant cette période inhibe l'activité de reproduction en mimant un jour long. Comme la stimulation par les jours courts ne fonctionne qu'après une période d'imprégnation suffisante par les jours longs (Legan et Karsch 1983), la reprise de l'activité ovarienne implique que les brebis soient soumises à cette alternance. Après la fin de la saison de reproduction, le flash lumineux est appliqué 17 heures après l'aube pendant 2 mois (jours longs) puis ensuite est déplacé entre $8 \mathrm{~h}$ et $12 \mathrm{~h}$ après l'aube (jours courts). Ce traitement est facile à mettre en place dans les élevages et permet d'avancer la période de reproduction à la fin du printemps (Thimonier 1981). Des études en cours devraient permettre d'évaluer l'intérêt économique de cette méthode lorsqu'elle est associée à des traitements hormonaux ou à l'effet bélier.

La mise en évidence du rôle de la mélatonine dans la perception par le cerveau de la durée du jour, ouvre de nouvelles perspectives pour manipuler la reproduction chez la brebis. L'association d'un flash lumineux et d'implants de mélatonine est actuellement expérimentée sur le terrain (Chemineau et al 1988). L'administration de cette hormone pendant 4 semaines à l'aide d'implants sous cutanés, avance le début de la saison de reproduction des brebis adultes (Staples et al 1986) et la puberté chez les agnelles (Moore et al 1984).

\section{3 / Augmenter le taux d'ovulation}

La prolificité des brebis peut être améliorée en manipulant le taux d'ovulation par des approches très différentes (Scaramuzzi et Radford 1983). Dans les méthodes physiologiques modifiant le taux d'ovulation, on peut inclure l'utilisation des gonadotropines ou, plus récemment, l'utilisation d'implants de mélatonine (Staples et al 1986). Cependant, les progrès les plus importants effectués dans ce domaine sont liés à la mise au point de techniques d'immunisation contre les stéroïdes.

\section{1 / Immunisation contre les stéroïdes}

L'immunisation active ou passive contre les stéroïdes modifie la rétroaction ovarienne négative exercée sur l'axe hypothalamo-hypophysaire et augmente le taux d'ovulation. Pour le moment, l'immunisation active (c'est-à-dire l'animal sécrétant lui-même l'anticorps contre l'immunogène injecté) est la méthode la plus simple à mettre en place dans la mesure où le niveau plasmatique d'anticorps est maîtrisé et reproductible.

Un conjugué d'androsténedione et d'albumine sérique humaine dans un adjuvant DEAE dextran (FecundinGLAXO, Pty Ltd) administré par voie sous-cutanée 2 fois, à 4 semaines d'intervalle, a permis d'obtenir en moyenne une augmentation de 0,60 ovulation par brebis immunisée (Smith 1985).

Lévaluation sur le terrain de cette nouvelle technique a été menée à grande échelle principalement en Australie (Geldard 1984) et en Nouvelle Zélande (Smith 1985) chez une grande diversité de races et de systèmes de production. L'augmentation maximum d'agneaux nés est observée lorsque la lutte commence 3 semaines après l'injection de rappel chez des brebis ayant une condition corporelle suffisante. Cette augmentation varie selon les génotypes étudiés : $18 \%$ en Mérinos, $31 \%$ en Border Leicester $\times$ Mérinos et $40 \%$ chez les Romney pures ou croisées (Geldard 1984).

Ces différences peuvent être dues à des réponses à l'immunisation variant avec le génotype et/ou au fait qu'une mortalité embryonnaire plus importante est associée à des titres d'anticorps élevés (Boland et al 1986).

Sur le plan pratique, il est intéressant de noter que chez les races ovines françaises étudiées, le gain d'agneaux par brebis traitée avec Fecundin est plus important lorsque le niveau naturel de prolificité est faible contre les stéroïdes augmente la prolificité des brebis, l'augmentation étant d'autant plus importante que le taux moyen de prolificité de la race est faible.
Tableau 7. Performances de reproduction obtenues chez des brebis de race Charmoise immunisées (I) ou non (C) pendant 4 années successives (Lycée agricole de Fondettes).

\begin{tabular}{|c|c|c|c|c|c|}
\hline \multirow{2}{*}{ Année } & Traitement & $(\mathrm{n})$ & Taux fertilité & Taux prolificité & $\begin{array}{c}\text { Nomb. d'agneaux } \\
\text { sevrés par } \\
\text { brebis luttée }\end{array}$ \\
\hline 83 & $\mathrm{C}$ & 23 & 1.00 & 1.09 & 1.04 \\
& $\mathrm{I}$ & 24 & 0.96 & 1.70 & 1.54 \\
84 & $\mathrm{C}$ & 22 & 0.91 & 1.05 & 0.96 \\
& $\mathrm{I}$ & 23 & 0.91 & 1.76 & 1.30 \\
85 & $\mathrm{C}$ & 21 & 1.00 & 1.29 & 1.14 \\
& $\mathrm{I}$ & 17 & 0.88 & 1.67 & 1.24 \\
& $\mathrm{C}$ & 16 & 0.94 & 1.27 & 1.13 \\
& $\mathrm{I}$ & 11 & 1.00 & 1.91 & 1.63 \\
\hline Total & $\mathrm{C}$ & 82 & 0.96 & 1.16 & 1.06 \\
4 années & $\mathrm{I}$ & 75 & 0.93 & $1.74 \quad \mathrm{P}<0.01$ & 1.39 \\
\hline
\end{tabular}


Tableau 8. Influence de la période de l'année sur la réponse à un traitement pFSH pour le transfert d'embryons chez la brebis Lacaune. (D'après Torres et al 1984).

\begin{tabular}{|l|c|c|c|c|c|}
\hline Période & $\begin{array}{c}\text { Nombre } \\
\text { de brebis } \\
\text { donneuses }\end{array}$ & $\begin{array}{c}\text { Taux } \\
\text { d'ovulation }\end{array}$ & $\begin{array}{c}\text { Nb d'embryons } \\
\text { récupérés par } \\
\text { donneuse }\end{array}$ & $\begin{array}{c}\text { Nb d'embryons } \\
\text { transférés par } \\
\text { donneuse (\%) }\end{array}$ & $\begin{array}{c}\text { Taux de fertilité* } \\
\text { des brebis } \\
\text { receveuses (\%) }\end{array}$ \\
\hline Saison sexuelle & 22 & $11.2 \pm 1.4$ & $9.3 \pm 1.4$ & $6.3 \pm 1.4(74.5)$ & 76.3 \\
Anœstrus saisonnier & 20 & $\mathrm{P}<0.05$ & $\mathrm{P}<0.05$ & $\mathrm{P}<0.05$ & $\mathrm{~ns}$ \\
\hline
\end{tabular}

* 2 embryons de 6 jours ont été transférés par brebis receveuse.

(Philipon et Driancourt 1987). Ce gain est maintenu après utilisation répétée de ce traitement (tableau 7) et est obtenu sans une augmentation significative des portées égales ou supérieures à trois (Cognié 1988). Ce dernier point, ajouté à des charges en main d'œuvre plus faibles, sont des avantages suffisamment importants pour orienter le choix vers les techniques d'immunisation plutôt que vers le traitement progestagène-PMSG, dans les zones de production extensive désirant améliorer la productivité des troupeaux en saison sexuelle.

Par contre, à l'inverse du traitement progestagènePMSG, l'immunisation contre les stéroïdes ne peut seule induire des ovulations chez les brebis en ancstrus. Dans cette période, il faut donc l'associer à l'effet mâle ou au traitement progestagène-PMSG (Robinson et Scaramuzzi 1986).

\section{2 / Manipulation de la sécrétion endogène de FSH}

La synthèse et la libération de FSH par l'hypophyse est freinée par une hormone peptidique, l'inhibine, qui est sécrétée par les cellules de la granulosa dans les follicules à antrum. Chez la brebis, l'élimination des gros follicules par électrocautérisation (Cognié et al 1986) ou par infusion de liquide folliculaire (Wallace et al 1985) est suivie d'une augmentation des niveaux plasmatiques de FSH et du taux d'ovulation.

L'immunisation active de brebis à l'aide d'une préparation de liquide folliculaire, enrichie en inhibine, augmente également le taux d'ovulation mais avec un coefficient de variabilité élevé (O'Shea et al 1984).

\section{3 / Administration exogène de FSH pour le transfert d'embryons}

Le transfert d'embryons, après superovulation des brebis donneuses, permet d'accélérer le progrès génétique (Smith 1986) et facilite la production d'animaux transgéniques (Warld et al 1984). Le transfert d'embryons n'est pas couramment utilisé à cause de la difficulté à obtenir un nombre élevé d'embryons de bonne qualité après traitement par PMSG. L'injection de 1200 à 1500 UI de PMSG entraîne un développement folliculaire excessif, un environnement utérin défavorable et la dégénérescence d'une grande partie des embryons chez la brebis traitée. En utilisant une préparation de FSH porcine ( $\mathrm{pFSH}$ ) et un traitement séquentiel (injections matin et soir pendant 2 jours) on évite ces inconvénients. Cette préparation permet de mimer la séquence hormonale naturelle observée pendant la fin du cycle en injectant la FSH à des doses décroissantes et la LH à des doses croissantes. En moyenne, 7,5 et 5,9 embryons transférables par donneuse sont obtenus chez des brebis llede-France traitées avec $16 \mathrm{mg}$ de pFSH respectivement en saison ou hors saison de reproduction (Cognié et al
1986). La réponse à pFSH varie en fonction du génotype (elle est plus élevée chez les races naturellement prolifiques) et de l'époque de l'année (tableau 8).

\section{4 / Réduire la mortalité embryonnaire et la mortalité des agneaux}

\section{1 / Réduction de la mortalité embryonnaire}

La mortalité embryonnaire est un déterminant important des taux de fertilité et de prolificité et peut être attribuée, soit à des anomalies génétiques, soit à un milieu utérin défavorable au développement de l'embryon. Il paraît difficile d'échapper aux premières sources de pertes $(11 \%$ des embryons présentent des anomalies léthales, Boland et al 1986), par contre on a essayé de limiter les secondes au moyen de diverses interventions thérapeutiques.

Il est établi que la mortalité embryonnaire survient principalement au cours du premier mois de la gestation (Edey 1969) et augmente dans les situations suivantes :

- ovulations multiples après un œstrus naturel (White et al 1981), ou induit par traitement hormonal (Lunstra et Christenson 1981) ou immunisation contre l'androsténedione (Boland et al 1986).

- induction hormonale de l'œstrus pendant la période prépubère (Quirke et Hanrahan 1977) ou pendant l'anœstrus de lactation (Cognié et al 1975).

Dans ces études, l'augmentation de la mortalité embryonnaire est souvent associée à un retard de développement des embryons. L'administration de progestérone à des brebis, 5 à 15 jours après la saillie, afin de réduire les pertes embryonnaires a donné des résultats favorables (Pearce et al 1984) ou non (Smith et al 1985). Les interactions entre le niveau nutritionnel des brebis traitées et le métabolisme de la progestérone peuvent expliquer en partie l'inconsistance de cette thérapie (Parr et al 1986).

Une injection de 100 UI de HCG (Human Chorionic Gonadotrophin) $12 \pm 1$ jours après la saillie augmente la sécrétion de progestérone et le taux de fertilité chez les brebis allaitantes traitées (Kittock et al 1983). Dans une expérience menée en France à grande échelle, les brebis ayant reçu une injection de 300 UI de PMSG 15 jours après l'IA, ont un taux de prolificité plus élevé (181\% vs $165 \%, \mathrm{P}<0.05)$ mais un taux de fertilité identique (57\%) à celui des témoins non traitées (ITOVIC, INTERVET, non publié).

Ces résultats montrent que le niveau de mortalité embryonnaire précoce peut être diminué (et semble-t-il d'autant plus que les paramètres de reproduction sont faibles chez les témoins) en manipulant les niveaux plasmatiques de progestérone au moment où l'embryon se fixe sur la paroi utérine. 


\section{2 / Diagnostic de gestation et amélioration de la conduite des brebis pendant la gestation}

La connaissance du nombre de brebis gestantes après induction des chaleurs hors de la saison normale de reproduction et du nombre de fotus portés par les mères, permet à l'éleveur d'une part de remettre rapidement les brebis non gestantes en lutte et d'autre part de mieux préparer les mères à l'agnelage.

Durant les dix dernières années, le diagnostic précoce de gestation basé sur l'estimation des niveaux plasmatiques de progestérone $18 \pm 1$ jours après la lutte (Thimonier et al 1977) a été largement utilisé, tout au moins dans notre pays. Cette méthode est très précise pour détecter les brebis non fécondées mais seulement $90 \%$ environ des brebis diagnostiquées gestantes mettent bas. La proportion de "fausses gravidités" varie selon les troupeaux et l'état physiologique des brebis : elle est respectivement en moyenne de $13 \%, 7 \%$ et $8 \%$ chez les femelles allaitantes, taries ou prépubères, et reflète l'importance des pertes embryonnaires chez ces femelles. Bien que la méthode soit peu utilisée en routine, le nombre de fœetus peut être évalué aux environs du $100^{\mathrm{e}}$ jour de gestation en mesurant les œstrogènes totaux ou l'hormone placentaire lactogène dans le sang maternel (Thimonier et al 1977).

Dans un futur proche, ces méthodes biochimiques seront probablement remplacées par les techniques utilisant les ultrasons, plus simples d'emploi. La mise en évidence du flux sanguin fœtal par effet Doppler ou la détection de la présence de liquide amniotique dans l'utérus par échoscopie sont des méthodes utilisables avec succès pendant la deuxième moitié de la gestation mais elles ne permettent pas une estimation de la taille de la portée (Jardon et al1984).

Lemploi de l'échographie (real time ultrasound scanning) permet de détecter, à l'aide d'une sonde rectale, la présence du foetus dès le $23^{\mathrm{e}}$ jour de la gestation. Avec une sonde externe, il est possible de déterminer avec précision l'état de gravidité et le nombre de fœetus $(1$, 2 ou plus de 2 ) dès le $40^{\circ}$ jour de la gestation. En dépit du coût élevé de l'échographe, cette méthode a été rapidement acceptée du fait de sa simplicité, de sa rapidité ( 1000 diagnostics par jour par une équipe de 3 personnes) et des bénéfices que l'on peut en tirer. En effet, l'adaptation des apports nutritionnels en fin de gestation au nombre de foetus permet une amélioration significative du taux de survie et de la croissance des agneaux nés (Fowler et Thompson 1986).

Le dosage du glucose sanguin, au milieu de la gestation, permet d'identifier les brebis nécessitant un apport d'aliments supplémentaire. La supplémentation des brebis hypoglycémiques peut réduire dans des conditions d'élevage extensif (Parr et al 1986a) la mortalité périnatale des agneaux.

\section{3 / Contrôle de l'agnelage}

Les agnelages des brebis synchronisées sont normalement groupés dans une période de 7 à 10 jours. Une injection de $16 \mathrm{mg}$ de dexaméthasone 4 jours avant la fin de la durée moyenne de gestation induit la mise-bas dans les 48 à 96 heures (Bosc 1972). En système intensif, cette méthode de groupage des mises-bas a permis une réduction significative de la mortalité des agneaux, surtout chez les primipares (Cognié et al 1980). Cependant son utilisation ne peut être envisagée que lorsque un nombre suffisant de cases d'adoption (isolation des

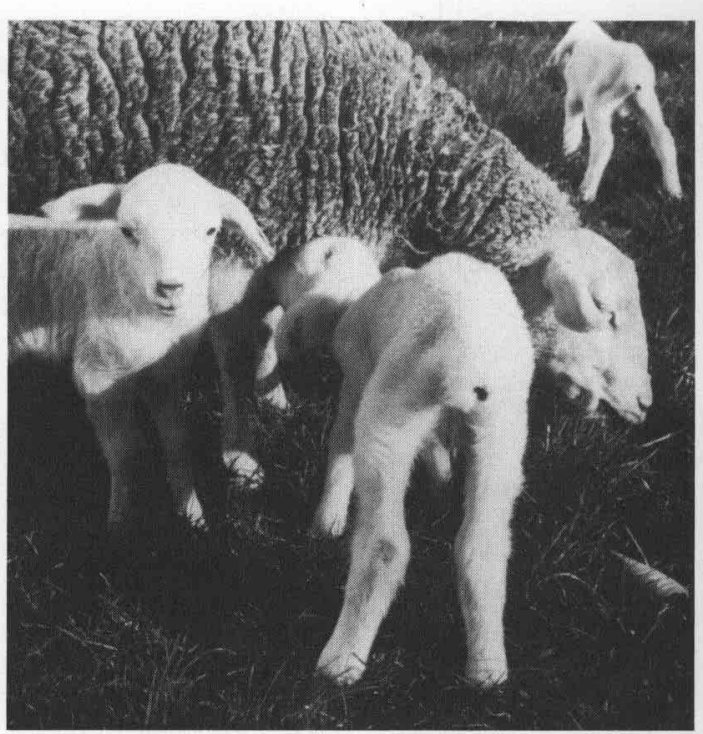

mères de doubles ou des mères ayant un comportement maternel peu développé) est prévu dans la bergerie.

Linjection de 15 à $20 \mathrm{mg}$ de benzoate d'œstradiol est une méthode peu onéreuse pour synchroniser les agnelages. Elle a l'avantage de favoriser la lactation et le comportement maternel (Poindron et al 1984). Il faut cependant être conscient que l'injection prématurée d'œstrogènes (avant la dernière semaine de gestation) entraîne l'avortement des brebis traitées, ce qui ne se produit pas lorsqu'on utilise la dexaméthasone à mauvais escient.

\section{$5 /$ Accélération du rythme d'agnelage}

Différents systèmes permettant une production d'agneaux tout au long de l'année et intégrant à un degré variable les nouvelles technologies évoquées dans cet article, ont été proposées dans différentes régions du monde (Gordon 1983). Le succès commercial d'une telle entreprise peut être plus facilement atteint quand :

- le génotype utilisé a un niveau élevé de fécondité : croisements Finnois et Romanov (Nord de l'Europe, USA, Canada) ou lignées synthétiques (Hyfer en Australie, INRA 401 en France). Lorsqu'on utilise une race ayant une longue période d'ancestrus saisonnier, l'augmentation de productivité obtenue avec l'accélération du rythme d'agnelage ne suffit pas à compenser les coûts de main-d'œuvre supplémentaires et des traitements hormonaux (Cognié et al 1980).

- les apports alimentaires sont adaptés de façon à ce que les réserves corporelles mobilisées en fin de gestation et début de lactation soient reconstituées avant la remise en lutte des brebis (Robinson et al 1979).

- l'intervalle entre l'agnelage et la mise en place du traitement progestagène-PMSG est soigneusement évalué en fonction du génotype et de l'époque de l'année, de façon à minimiser la mortalité embryonnaire (Tableau 4).

Dans ces conditions, 3,0 et 3,8 agneaux/brebis/an ont été produits pendant plusieurs années successives respectivement par des croisées Romanov (Thimonier et Cognié 1977) et des Romanov (Cornu et Cognié 1983). L'utilisation de rythmes lumineux inversés (ou d'implants de mélatonine) pour réduire les variations saisonnières des performances de reproduction permet d'augmenter encore davantage la production d'agneaux (Ainsworth et Shrestha 1985). L'introduction de l'immunisation contre les stéroïdes dans des systèmes de pro- 
duction intensive est en cours d'évaluation et les premiers résultats semblent favorables (Cognié 1988).

\section{Conclusion}

Pour un nombre de plus en plus important d'éleveurs, la brebis élevant 2 agneaux par an est devenue une réalité. Certains envisagent même d'atteindre un niveau de productivité plus élevé. Différentes stratégies pour atteindre ce but ont été développées dans cet article ; mais le choix d'une stratégie par l'éleveur est avant tout guidé par son environnement économique. La première étape, avant d'utiliser des méthodes sophistiquées pour améliorer l'efficacité de la reproduction, est d'arriver à une bonne maîtrise de la conduite du troupeau en particulier sur les plans pathologique et nutritionnel. Dans une région difficile comme celle de l'Australie de l'Ouest, deux types d'interventions peu couteuses sont mises en place de façon prioritaire

1. la distribution de suppléments azotés (lupin) pour améliorer les performances de reproduction des brebis et des béliers au cours de la lutte de printemps,

2. l'utilisation de l'effet bélier pour mieux désaisonner l'activité sexuelle des brebis.

Dans nos régions plus favorisées, l'adoption par les éleveurs du traitement progestagène-PMSG illustre comment une technique convenablement utilisée peut contribuer à l'amélioration de la productivité des troupeaux. D'autres techniques décrites dans cette revue vont dans un proche avenir être proposées aux éleveurs pour manipuler le taux d'ovulation (immunisation contre les stéroïdes) ou l'aptitude au désaisonnement (implants de mélatonine). Ces techniques doivent d'abord être évaluées en fonction du matériel génétique utilisé et des systèmes de production particuliers à chaque région afin de juger de leur intérêt économique.

Laugmentation du taux d'ovulation après immunisation contre les stéroïdes ou après utilisation du traitement progestagène-PMSG augmente la taille de la portée mais également le taux de fertilité chez les brebis traitées. Le prix à payer pour améliorer la fertilité du troupeau est donc d'être capable d'élever davantage d'agneaux nés de portées multiples.

L'augmentation du taux d'ovulation par thérapie hormonale peut avoir des conséquences non désirées et parfois accroitre les pertes embryonnaires précoces. Pour limiter ces effets défavorables et tirer un meilleur parti de ces nouvelles méthologies, les conditions d'environnement hormonal permettant un développement optimal du jeune embryon doivent être précisées.

\section{Références bibliographiques}

AINSWORTH L., SHRESTHA J.N.B., 1985. Theriogenology 24 479-487.

BINDON B.M., PIPER L.R., CAHILL L.P., DRIANCOURT M.A., O'SHEA T., 1986. Theriogenology 25, 53-70.

BOLAND M.P., MURRAY J.D., SCARAMUZZI R.J., NANCARROW C.D., HOSKINSON R.M., SUTTON R., HAZELTON I., 1986. In : Embryonic Mortality in farm animals. Eds Sreenan and Diskin, pp. 171-178. Martinus Nijhoff publishers for the CEC

BOSC M.J., 1972. J. Reprod. Fert. 28, 347-357.

BRICE G., CACHENAUT J.B., COGNIÉ Y., ROUSSELY M., SALAUN J., 1984. IX journées de la recherche ovine et caprine. Ed. INRAITOVIC - SPEOC, Paris, 134-151.

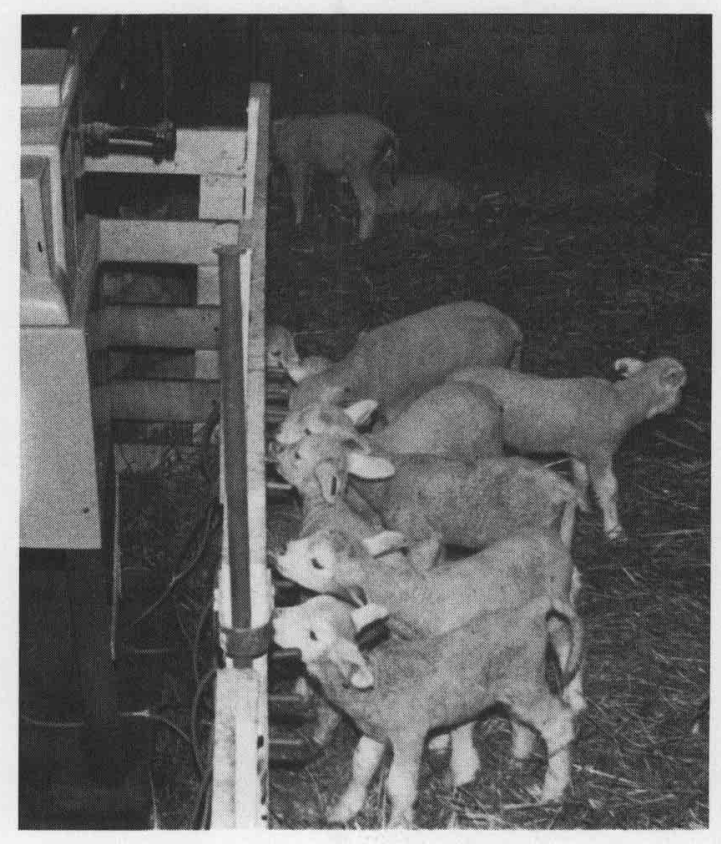

\section{Augmenter la productivité du troupeau nécessite d'élever davantage d'agneaux nés de portées multiples.}

CHEMINEAU P., PELLETIER J., GUÉRIN Y., COLAS G., RAVAULT J.P., TOURÉ G., ALMEIDA G., THIMONIER J., ORTAVANT R., 1988. Reprod. Nutr. Dévelop., 28, 2, 65-78.

COGNIÉ Y., 1988. XI Int. congr. anim. reprod. and A.I. Dublin, 25-30 juin, (sous presse).

COGNIÉ Y., MAULÉON P., 1983. In : Sheep production. Ed. W. Haresign, pp. 381-392. Butterworths, London.

COGNIÉ Y., MARIANA J.C., THIMONIER J., 1970. Ann. biol. an. bioch. bioph. 10, 15-24.

COGNIÉ Y., CORNU C., MAULÉON P., 1974. Proc. int. symp. on physiopathology of reproduction and Al in small ruminants. Thessaloniki 17-19 may.

COGNIÉ Y., HERNANDEZ-BARRETO M., SAUMANDE J., 1975. Ann. biol. an. bioch. bioph. 15, 329-343.

COGNIÉ Y., PERRET G., OLDHAM C.M., 1980. Proc. aust. soc. anim. prod. $13,305-308$.

COGNIÉ Y., SCHIRAR A., MARTINET J., POULIN N., MIRMAN B., 1986. IX journées de la recherche ovine et caprine. Ed. INRAITOVIC-SPEOC, Paris, pp. 109-133.

COGNIÉ Y., CHUPIN D., SAUMANDE J., 1986. Theriogenology 1, 148 (Abstr.).

COGNIÉ Y., SCARAMUZZI R.J., CAMPBELL B.K., DOWNING I.A., TURNBULL K.E., 1986. Proc. endocr. soc. aust. 29 (Abstr.).

COLAS G., 1983. In : Sheep production. Ed. W. Haresign, pp. 453-465. Butterworths, London.

COLAS G., GUÉRIN Y., 1979. V journées de la recherche ovine et caprine. Ed. INRA-ITOVIC-SPEOC, Paris, pp. 162-184.

COLAS G., THIMONIER J., COUROT M., ORTAVANT R., 1973. Ann. zootech. 22, 441-451.

COLAS G., GUÉRIN Y., CLANET V., ROQUES I.M., ALBERIO R., 1984. IX journées de la recherche ovine et caprine. Ed. INRAITOVIC-SPEOC, Paris, pp. 79-99.

COLAS G., GUÉRIN Y., CLANET V., SOLARI A., 1985. Rep. nut. dev. 25 (1A), 101-111.

CORNU C., COGNIÉ Y., 1983. In : The genetics of reproduciton in sheep. Eds. R.B. Land and D.W. Robinson, pp. 383-389. Butterworths, London.

DUTT R.H., CASIDA L.E., 1948. Endocrinology 43, 208-217. EDEY T.N., 1969. Anim, breed. abstr. 37, 173-190.

FOLCH J., COGNIÉ Y., SIGNORET J.P., 1985. Proc. sheep and goat production E.A.A.P. 30 sept. - 3 oct. Thessaloniki.

FUKUI Y., KOBAYASHI M., KOSIMA M., ONO H., 1985. Theriogenology 24, 631-641.

FOWLER D.G., THOMPSON J.A., 1986. Proc. aust. soc. anim. prod. 16, 410 (Abstr.).

GELDARD H., 1984. Proc. aust. soc. anim. prod. 15, 185-191. 
GORDON I., 1983. In : controlled breeding in farm animals. Part II. Chapters 10-20. Pergamon, Oxford.

HULET C.V., STORMSHAK F., 1972. J. Anim. sci. 34, 1011-1019. JARDON C., DE MONTIGNY G., ANDRÉ D., CORTELL J.M., BARIL G., COGNIÉ Y., BOTERO O., HUMBLOT P., 1984. IX journées de la recherche ovine et caprine. Ed. INRA-ITOVIC-SPEOC, Paris, pp. 452-473.

KITTOCK R.J., STELLFLUG J.N., LOWRY S.R., 1983. J. anim. sci., $56,652-655$.

LEGAN S.J., KARSCH FJ., 1983. Biol. reprod. 29, 316-325.

LINDSAY D.R., COGNIÉ Y., PELLETIER J., SIGNORET J.P., 1975. Phys. and Beh. 15, 423-426.

LUNSTRA D.D., CHRISTENSON R.K., 1981. J. anim. sci. 53, 458-466.

MAXWELL W.M.C., 1984. In : Reproduction in sheep. Eds D.R. Lindsay and D.T. Pearce, pp. 291-300. Australian academy of science.

MOORE R.W. MILLER C.M., LYNCH P.R., WELCH R.A.S., BAR NES D.R., HOCKEY H.U.P., 1984. Proc. N.Z. Soc. anim. prod. 44 21-23.

OLDHAM C.M., COGNIÉ Y., 1980. Proc. aust. soc. anim. prod. $13,82$.

OLDHAM C.M., MARTIN G.B., 1978. Anim. reprod. sci. 1, 291. OLDHAM C.M., PEARCE D.T., 1984. Proc. aust. soc. anim. prod. $15,158-170$

ORGEUR P., SIGNORET J.P., 1985. Phys. and beh. 33, 111.

ORTAVANT R., 1977. Proc. symposium on the management of reproduction in sheep and goats, pp. 58-71. Madison, Wisconsin. ORTAVANT R., PELLETIER J., RAVAULT J.P., THIMONIER J., VOLAND-NAIL P., 1985. Oxford Rev, reprod. biol., 7, 305-345. O'SHEA T., AL-OBAIDI S.A.R., HILLARD M.A., BINDON B.M., CUMMINS L.J., FINDLAY ].K., 1984. In : Reproduction in sheep. Eds D.R. Lindsay and D.T. Pearce, pp. 335-337. Australian academy of science.

PARR R.A., MILES M.A., LANGDON P.J., 1986a. Proc. aust. soc. anim. prod. 16, 299-302.

PARR R.A., DAVIS I.F., FAIRCLOUGH R.J., PILATTI E.M., GRAZEBROOK TW., HOLLAND G.F., 1986b. Proc. aust. soc. anim. prod. 16, 425. (Abstr.).

PEARCE D.T., GRAY S.J., OLDHAM C.M., WILSON H.R., 1984. Proc. aust. soc. anim. prod. 15, 164-168.

PERRET G., ROUSSELY M., 1984. IX journées de la recherche ovine et caprine. Ed. INRA-ITOVIC-SPEOC, Paris, pp. 34-61.
PHILIPON P., DRIANCOURT M.A., 1987. An. reprod. sci., 15, 101-112.

POINDRON P., LE NEINDRE P., LÉVY F, 1984. In : Reproduction in sheep. Eds D.R. Lindsay and D.T. Pearce. pp. 191-198. Australian academy of science.

QUINLIVAN T.D., ROBINSON T.J., 1969. J. reprod. fert. 19, 73-86. QUIRKE J.F., HANRAHAN J.P., 1977. J. reprod. fert. 51, 487-489. QUIRKE J.F., JENNINGS J.J., HANRAHAN J.P., GOSLING J.P., 1979. J. reprod. fert. $56,479-488$.

ROBINSON J.J., MCHATTIE I., CALDERON C.J.F., THOMPSON I.L., 1979. Anim. prod. 29, 257-269.

ROBINSON T.J., 1951. J, agric. sci. 41, 6-63.

ROBINSON T.J., 1964. Proc. aust. soc. anim. prod. 8, 47-49.

ROBINSON T.J., 1976. Proc. int. sheep breeding congr. (Muresk), pp. 509-523.

ROBINSON T.J., SCARAMUZZI R.J., 1986. Proc. aust. soc. anim. prod. 16, 323-326.

SCARAMUZZI R.J., RADFORD H.M., 1983. J. reprod. fert., 69, 353-367.

SMITH J.F., 1985. Proc. N.Z. Soc. anim. prod. 45, 171-177.

SMITH C., 1986. Anim. prod. 42, 81-88.

SMITH J.F., FARQUHAR P.A., WELCH R.A.S., 1985. Proc. endocr. soc. aust. 28 (Suppl. 2), 9 (Abstr.).

STAPLES L., FOOTE M., JONES A., MCPHEE S., REEVE J., WILKINS J., WILLIAMS A., 1986. Proc. aust. soc. reprod. biol., 18, 23 (Abstr.).

THIMONIER J., 1981.-. reprod. fert. suppl. 30, 33-45.

THIMONIER I., COGNIÉ Y., 1977. In : Management of Reproduction in sheep and goats, pp. 109-118. Madison, Wisconsin.

THIMONIER J., BOSC M., DIIANE J., MARTAL J., TERQUI M., 1977. Proc. symposium on the management of reproduction in sheep and goats, pp. 79-88. Madison, Wisconsin.

TORRES S., COGNIÉ Y., COLAS G., 1984. IX journées de la recherche ovine et caprine. Ed. INRA-ITOVIC-SPEOC, Paris, pp. 215-239. WARD K.A., MURRAY J.D., NANCARROW C.D., BOLAND M.P., SUTTON R., 1984. In : reproduction in sheep. Eds D.R. Lindsay and D.T. Pearce, pp. 279-285. Australian academy of science.

WELCH R.A.S., 1983. Proc. endocr. soc. aust. 26 (Suppl. 2), 46 (Abstr.).

WHITE D.M., RIZZOLI D.J., CUMMING I.A., 1981. Aust J. exp. agric. anim. husb. $21,32-38$. 